\title{
Conservación
}

\section{Estimación poblacional del cocodrilo americano (Crocodylus acutus) en el Parque Nacional Lagunas de Chacahua, Oaxaca, México}

\author{
Population estimate of American crocodile (Crocodylus acutus) in Lagunas de Chacahua National \\ Park, Oaxaca, Mexico \\ Jesús García-Grajales ${ }^{\mathrm{a}, *}$ y Alejandra Buenrostro-Silva ${ }^{\mathrm{b}}$ \\ ${ }^{a}$ Instituto de Recursos, Universidad del Mar, campus Puerto Escondido, Km. 2.5, Carretera Federal Puerto Escondido - Sola de Vega, 71980, San Pedro Mixtepec, \\ Oaxaca, México \\ ${ }^{\mathrm{b}}$ Instituto de Industrias, Universidad del Mar, campus Puerto Escondido, Km. 2.5, Carretera Federal Puerto Escondido - Sola de Vega, 71980 , San Pedro \\ Mixtepec, Oaxaca, México
}

Recibido el 18 de agosto de 2016; aceptado el 13 de junio de 2017

Disponible en Internet el 1 de diciembre de 2017

\begin{abstract}
Resumen
El estudio se realizó entre noviembre de 2013 y junio de 2014 con el objetivo de estimar la población del cocodrilo americano (Crocodylus acutus) en el Parque Nacional Lagunas de Chacahua (PNLCh). Se realizaron recorridos mensuales nocturnos durante 3 noches consecutivas en 3 localidades. Las tasas de encuentro registradas fluctuaron entre 2.3 (Zapotalito) a 33.4 (Palmarito) ind./km lineal. La localidad Palmarito presentó la estructura poblacional más representativa, mientras que en la localidad Zapotalito se registraron los porcentajes más bajos de avistamiento. Se encontraron diferencias significativas en la abundancia por mes de muestreo y por clase de edad en cuanto a la frecuencia de uso por ambientes, así como una interacción significativa entre el mes, el hábitat y la clase de edad. El tamaño poblacional total estimado por transecto fluctuó entre 2.8 y 47.9 individuos. La mayor abundancia de individuos se presentó en Palmarito (47.9 \pm 0.4 individuos), mientras que la más baja se encontró en Zapotalito ( $2.8 \pm 0.3$ individuos). Las primeras aproximaciones sobre la ecología poblacional y el estado actual del cocodrilo americano en el PNLCh son proporcionadas en este trabajo y representan la base del monitoreo poblacional en los años futuros para la administración de esta Área Natural Protegida.
\end{abstract}

(C) 2017 Universidad Nacional Autónoma de México, Instituto de Biología. Este es un artículo Open Access bajo la licencia CC BY-NC-ND (http://creativecommons.org/licenses/by-nc-nd/4.0/).

Palabras clave: Conservación; Monitoreo; Transectos; Conteo; Abundancia; Oaxaca

\section{Abstract}

This study was conducted from November 2013 to June 2014 with the aim of estimating the population of American crocodile (Crocodylus acutus) in the Lagunas de Chacahua National Park (PNLCh). The field work consisted in surveys during three consecutive nights each month on several localities. The recorded encounter rates (ER) obtained by transect lines ranged from 2.3 (Zapotalito) to 33.4 (Palmarito) ind $/ \mathrm{km}$. The locality Palmarito showed the most representative population structure, while Zapotalito presented the lowest percentage of sightings. Significant differences were found per month in the abundance with age class respect to the frequency of crocodile use of the environments, as well as a significant interaction between month, habitat and age class. The total population estimated by transect lines fluctuated between 2.8 and 47.9 individuals. The greater abundance of individuals occurred in Palmarito ( $47.9 \pm 0.4$ individuals), while the lowest was found in Zapotalito ( $2.8 \pm 0.3$ individuals). The first approximations on population ecology and the current status of the American crocodile in the PNLCh are provided in this research and represent the basis of population monitoring in future years for the administration of this Mexican protected site.

(C) 2017 Universidad Nacional Autónoma de México, Instituto de Biología. This is an open access article under the CC BY-NC-ND license (http://creativecommons.org/licenses/by-nc-nd/4.0/).

Keywords: Conservation; Monitoring; Transects; Count; Abundance; Oaxaca

\footnotetext{
* Autor para correspondencia.

Correo electrónico: archosaurio@yahoo.com.mx (J. García-Grajales).

La revisión por pares es responsabilidad de la Universidad Nacional Autónoma de México.
} 


\section{Introducción}

Históricamente, las poblaciones de cocodrilos en México fueron sometidas a una intensa presión de cacería por la industria peletera, lo que produjo una disminución de sus poblaciones silvestres (Thorbjarnarson et al., 2006), con el consecuente establecimiento de una veda nacional en 1970 (Anónimo, 2000). Por lo que respecta al cocodrilo americano (Crocodylus acutus), una de las 3 especies de cocodrilianos en el país, esta se encuentra incluida en la categoría de vulnerable por la Unión Internacional para la Conservación de la Naturaleza (PonceCampos, Thorbjarnarson y Velasco, 2012), catalogada en el apéndice I de la Convención para el Comercio Internacional de Especies de Flora y Fauna Silvestres (CITES, por sus siglas en inglés) y sujeta a protección especial por la normatividad ambiental mexicana (NOM-059-2010; Diario Oficial de la Federación, 2011). Además, es una de las especies de cocodrilos con mayor cobertura de investigaciones en el país (García-Grajales y López-Luna, 2010), posiblemente por su amplia distribución geográfica (costas del golfo de México, el Caribe y Pacífico mexicano) (Ernst, Ross y Ross, 1999). Sin embargo, el conocimiento sobre el estado de sus poblaciones en México aún presenta vacíos geográficos de información (García-Grajales y López-Luna, 2010), específicamente en los estados de Michoacán, Guerrero y Oaxaca (García-Grajales y Buenrostro-Silva, 2014a).

En Oaxaca se han realizado trabajos sobre ecología y conservación de cocodrilos americanos que muestran datos poblacionales (Brandon-Pliego, 2007; García-Grajales y Buenrostro-Silva, 2014a, 2015; García-Grajales y EspinosaReyes, 2001). No obstante, aún existen zonas del estado en las que se desconoce la situación poblacional de la especie. Aunado a lo anterior, en la última década se han incrementado los registros de conflictos humanos-cocodrilos en los que, lamentablemente, han ocurrido lesiones físicas y decesos de personas (García-Grajales, Buenrostro-Silva y Brandon-Pliego, 2008; García-Grajales y Buenrostro-Silva, 2013) así como pérdida de animales domésticos (García-Grajales y Buenrostro-Silva, 2013); situación que se ha relacionado con el incremento de las poblaciones silvestres (García-Grajales y Buenrostro-Silva, 2015). Este tipo de conflictos ha estimulado que la población civil demande al gobierno estatal y federal la realización de acciones encaminadas al conocimiento de la abundancia de las poblaciones de cocodrilos en la costa del estado, principalmente las adyacentes a los asentamientos humanos, para definir áreas de riesgo potencial (García-Grajales y BuenrostroSilva, 2015).El decreto de creación del Parque Nacional Lagunas de Chacahua (PNLCh), Oaxaca, en 1937, lo coloca como una de las primeras áreas naturales protegidas del país. Es un sistema terrestre $(11,598 \mathrm{ha})$ y un sistema lagunar (3,324 ha) que proporciona un alto valor ecosistémico (García-Grajales y Buenrostro-Silva, 2014b). El cocodrilo americano es uno de los recursos naturales del Parque de importancia crucial para la conservación de sus ecosistemas (Semarnat, 2014). Sin embargo, al encontrarse en competencia con los asentamientos afromestizos por hábitat y los recursos pesqueros (García-Grajales y Buenrostro-Silva, 2015), provoca que los conflictos con los humanos se acentúen, por lo que resulta necesario conocer el estado poblacional de los cocodrilos para establecer acciones de manejo. El cocodrilo americano, al ser un depredador tope, es usado como indicador del estado de conservación de los ecosistemas en los que habita (Mazzotti, Best, Brandt, Cherkiss, Jeffrey y Rice, 2009). Además, se considera una especie focal para realizar el monitoreo de los ecosistemas marinos, costeros y de los humedales en las áreas naturales protegidas donde se distribuye (Venegas-Anaya, Escobedo-Galván, Balaguera, Lowrance, Sanjur y Densmore, 2015), lo que permite obtener información para generar el diseño, aplicación y evaluación de los planes de manejo para la conservación en este tipo de sitios (Venegas-Anaya et al., 2015). En este sentido, el monitoreo de largo plazo es una herramienta que permite conocer, hasta cierto punto, los impactos actuales y futuros, así como las fluctuaciones y cambios antropogénicos que ocurren en un área determinada (Venegas-Anaya et al., 2015).

Para lograr establecer las bases de un programa de monitoreo de cocodrilo de largo plazo en el PNLCh, este trabajo tiene por objetivo evaluar la abundancia y la estructura poblacional de la especie.

\section{Materiales y métodos}

El PNLCh se ubica en la porción central de la costa sur del estado de Oaxaca, en la región hidrológica RH-21, costa de Oaxaca y en colindancia con la región hidrológica RH-20, Costa Chica-Río Verde (Pérez-Delgado, 2002). Por las características geológicas se ubica en la provincia fisiográfica Costa Sur, que corresponde a un macizo terrestre angosto de $1,400 \mathrm{~km}$ de longitud, $25 \mathrm{~km}$ de ancho en la parte media y altitud promedio de $100 \mathrm{~m}$ snm, conformado de planicies costeras y lomeríos de baja a mediana altura (Pérez-Delgado, 2002).

El sistema de lagunas del PNLCh comprende 3,324 ha de cuerpos de agua entre los que se incluye el complejo Chacahua-Pastoría y otras 4 lagunas menores interconectadas por canales angostos: Salina Grande, Poza del Mulato, Poza de los Corraleños y Palizada (Pérez-Delgado, 2002). La vegetación predominante es el manglar (Rhizophora mangle, Laguncularia racemosa, Avicenia germinans y Conocarpus erectus) y con vegetación asociada a selva baja caducifolia, selva mediana subcaducifolia y subperennifolia, así como vegetación de zonas inundables (Torres-Colín, 2004).

El clima de la región es cálido subhúmedo (Aw1 (w) (i)) (García, 1988), con una temperatura media anual superior a los $28^{\circ} \mathrm{C}$, temperatura media máxima de $37^{\circ} \mathrm{C}$ y mínima de $23.2^{\circ} \mathrm{C}$. El mes más frío supera los $18^{\circ} \mathrm{C}$ con lluvias concentradas en el verano y principios del otoño, generalmente de julio a octubre; isoterma con una oscilación menor de $5{ }^{\circ} \mathrm{C}$. La precipitación anual es de aproximadamente 1,000 mm (Hernández-Santos, 2009; Marini, 1999).

Se realizaron recorridos mensuales nocturnos sistemáticos durante 3 noches consecutivas y durante la fase de luna nueva, en 2 equipos de trabajo entre noviembre de 2013 y junio de 2014. Para ello, seleccionamos 3 localidades (Las Salinas, Palmarito y Cerro Hermoso; fig. 1) con base en su accesibilidad, presencia y disponibilidad de hábitat para cocodrilos, así como su 


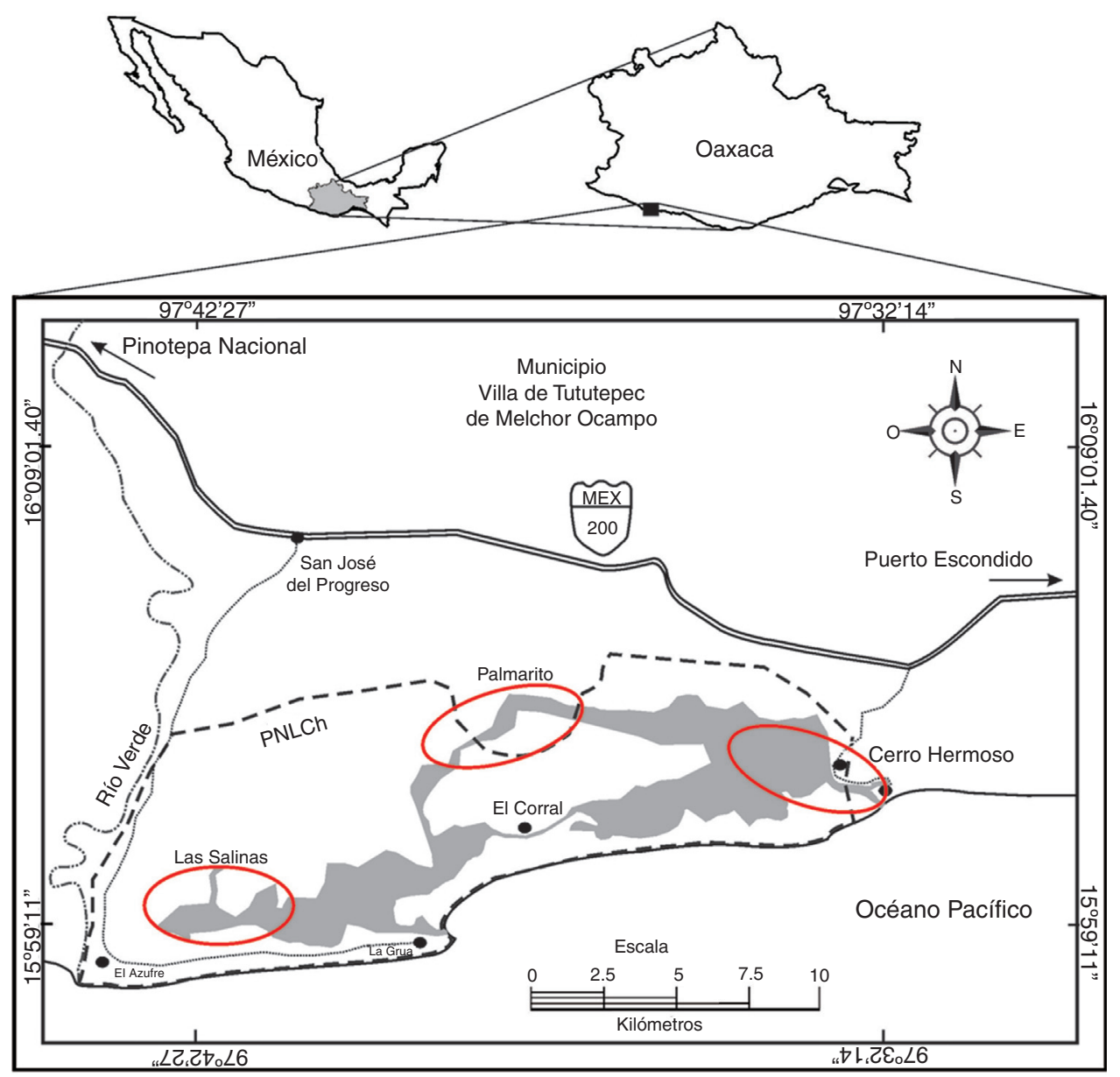

Figura 1. Ubicación de las localidades (óvalos en rojo) seleccionadas dentro del Parque Nacional Lagunas de Chacahua, Oaxaca.

representación espacial (Thorbjarnarson, 1989; Venegas-Anaya et al., 2015) dentro del Área Natural Protegida (ANP). En cada localidad establecimos 2 transectos fijos con longitudes variables entre sí (Las Salinas: transectos Vara quebrada y Agua dulce; Palmarito: transectos El Grueso y Palmarito; Cerro Hermoso: transectos rancho Viejo y Zapotalito) y dispuestos sobre el eje de los canales existentes en las lagunas; se recorrieron 2 transectos por equipo en cada noche de trabajo.

En cada recorrido nocturno se utilizaron 2 lanchas de madera (pangas) de fondo plano de $3.5 \mathrm{~m}$ de largo, propulsadas con motores fuera de borda de $15 \mathrm{hp}$ a una velocidad de $10 \mathrm{~km} / \mathrm{h} \mathrm{o}$ mediante el uso de remos, dependiendo de la amplitud y profundidad de los transectos, empleando para ello un esfuerzo de 4 personas por equipo de trabajo. Los recorridos se realizaron de manera sistemática a partir de las $22 \mathrm{~h}$ y hasta concluir el transecto establecido. Por cada muestreo contabilizamos los cocodrilos para determinar la tasa de encuentro (TE) [número de individuos (ind.) $/ \mathrm{km}$ lineal] a través del método descrito por Chabreck (1966), que consiste en el conteo visual nocturno, ubicando a los organismos por el destello de sus ojos a través del reflejo de un haz de luz con ayuda de lámparas de 6 volts y reflectores (spot line) de un millón de candelas. Asimismo, se estimó la separación de los ojos, así como la distancia de estos a la punta del hocico con la finalidad de determinar visualmente la longitud total (LT) de cada organismo (Messel, Vorlicek, Wells y Green, 1981; Thorbjarnarson, 1989).

Los organismos fueron clasificados, siguiendo los criterios de Thorbjarnarson (1989), como clase I ( $L T=<30 \mathrm{~cm}$ a $<60 \mathrm{~cm}$, neonatos y crías), clase II ( $L T=61$ a $120 \mathrm{~cm}$, juveniles), clase III ( $\mathrm{LT}=121-180 \mathrm{~cm}$; subadultos), clase IV (LT $=181$ $240 \mathrm{~cm}$, adultos), clase V $(\mathrm{LT}=>240 \mathrm{~cm}$, adultos reproductores) y solo ojos (SO, longitud no determinada) cuando no fue posible determinar el tamaño. Además, se relacionó el sitio donde se encontraba el ejemplar observado y el hábitat al momento de su observación (en el agua, bajo el manglar y en la periferia, en las orillas de la laguna desprovista de vegetación o en las orillas asociadas a la arena de la playa) con el fin de cuantificar la frecuencia de uso por ambientes al momento del conteo (García-Grajales y Buenrostro-Silva, 2014a).

Para estimar el tamaño poblacional se utilizó el modelo de Messel et al. (1981), modificado por Thorbjarnarson, Platt y Saw-Tun-Khaing (2000), que consiste en calcular el valor porcentual de la población observada $(\mathrm{P})$, a partir de la cual se estima la población total $(\mathrm{N})$. Con lo anterior se obtuvo una 
Tabla 1

Tasa de encuentro y estimación poblacional de Crocodylus acutus en el Parque Nacional Lagunas de Chacahua, Oaxaca.

\begin{tabular}{|c|c|c|c|c|}
\hline Localidad & Transecto & Longitud del transecto & TE (ind./km lineal) & $\mathrm{N} \pm \mathrm{DE}$ \\
\hline \multirow[t]{2}{*}{ Las Salinas } & Varaquebrada & $0.8 \mathrm{~km}$ & 12.5 & $9.7 \pm 0.4$ \\
\hline & Agua dulce & $1.1 \mathrm{~km}$ & 16 & $14.9 \pm 0.6$ \\
\hline \multirow[t]{2}{*}{ Palmarito } & El Grueso & $1.1 \mathrm{~km}$ & 7 & $6.3 \pm 0.2$ \\
\hline & Palmarito & $1.7 \mathrm{~km}$ & 49 & $47.9 \pm 0.4$ \\
\hline \multirow[t]{2}{*}{ Cerro Hermoso } & Rancho viejo & $1 \mathrm{~km}$ & 13 & $12.3 \pm 0.4$ \\
\hline & Zapotalito & $0.9 \mathrm{~km}$ & 4 & $2.8 \pm 0.3$ \\
\hline
\end{tabular}

DE: desviación estándar; N: estimación poblacional; TE: tasa de encuentro.

estimación de la densidad total mediante el recuento directo de los cocodrilos observados.

La estimación de la fracción de la población observada se calculó como sigue (Messel et al., 1981):

$$
P=\frac{m}{(2 s+m) 1.05}
$$

donde $\mathrm{P}$ es el porcentaje de la población observada, $\mathrm{m}$ es la media del número de cocodrilos observados en el total de los muestreos, s es la desviación estándar del número de cocodrilos observados para el total de los muestreos, y el nivel de error es de 1.05 .

El cálculo de la estimación total de la población $(\mathrm{N})$, con un nivel de confianza del 95\%, aceptando la normalidad de los conteos (Messel et al., 1981), es:

$N=\frac{m}{p}+\frac{[1.96(s)] 1 / 2}{(2 s+m) 1.05}$

donde 1.96 es el valor crítico tomado de $\mathrm{F}$ al $95 \%$ de confiabilidad.

El modelo de Messel et al. (1981) asume normalidad en los datos de conteo de cocodrilos (Cupul-Magaña, 2009); no obstante, se aplicó la prueba Shapiro-Wilk (Zar, 2010) a los datos con la finalidad de corroborar dicha normalidad.

Para establecer la existencia significativa de una relación entre la frecuencia de uso por ambientes por clase de tamaño, se realizó un análisis de varianza (Andeva) de 3 vías (mes muestreado, hábitat donde se observó al cocodrilo y clase de edad por talla). El cálculo de la clase de edad por talla se obtuvo del porcentaje de organismos observados en cada transecto. Posteriormente, esta variable se transformó con la función arcoseno (Zar, 2010), ya que las variables expresadas en porcentaje no presentan función normal.

Por otro lado, para detectar diferencias significativas entre el tamaño total de la población calculada por transecto, se aplicó una prueba de bondad de ajuste con un nivel de significación del $5 \%$.

\section{Resultados}

En total se obtuvieron 937 registros visuales de cocodrilos durante 48 recorridos, efectuados en $52.8 \mathrm{~km}$ durante 24 noches de trabajo. Las TE registradas fluctuaron de 2.3 (transecto Cerro Hermoso) a 33.4 (transecto Palmarito) ind./km lineal; con una TE promedio de 49 ind./km lineal (tabla 1).
La estructura de la población por localidad muestra que el transecto Palmarito presentó los porcentajes más altos respecto al avistamiento de ejemplares de la clase V (2.5\%), IV (15.2\%), III (21.4\%) y II (6.7\%), seguidos del transecto Agua dulce (0.9, $5.7,14.8$ y $5.3 \%$ ) en el mismo orden de clases. El transecto Zapotalito presentó los porcentajes más bajos de avistamiento. En este trabajo no se observaron organismos de la clase I en ninguno de los transectos recorridos (fig. 2). El tamaño poblacional total estimado por transecto fluctuó entre 2.8 y 47.9 individuos. De manera general, la mayor abundancia de individuos se presentó en el transecto Palmarito ( $47.9 \pm 0.4$ individuos) de la localidad del mismo nombre, mientras que la más baja se encontró en el transecto Zapotalito ( $2.8 \pm 0.3$ individuos) de la localidad de Cerro Hermoso. Estos resultados fueron significativamente diferentes (Chi cuadrada $=56.43$, g.l. $=11, p<0.05$ ) entre todas las localidades. Temporalmente, la mayor abundancia se registró en el mes de enero en el transecto Palmarito $(74.9 \pm 0.5$ individuos) y la menor en el mes de marzo en el transecto Zapotalito (1.9 \pm 0.3 individuos) (fig. 3 ).

En cuanto a la frecuencia de uso por ambientes al momento de los conteos, se encontraron diferencias significativas en la abundancia por mes de muestreo (Andeva: $\mathrm{F}_{29,97}=2.86, p<0.05$ ) $\mathrm{y}$ por clase de edad (Andeva: $\mathrm{F}_{6,86}=15.497, p<0.05$ ). Además, se comprobó la existencia de una interacción significativa entre los 3 factores: mes, hábitat y clase de edad (Andeva: $\mathrm{F}_{48,97}=2.714, p<0.05$ ), demostrando que la actividad de los organismos depende del tamaño y mes de muestreo. Los adultos reproductores (clase V) y adultos (clase IV) fueron observados en mayor proporción nadando en el agua (3 y 15\%, respectivamente), mientras que los subadultos (clase III) y juveniles (clase II) fueron observados entre las raíces del manglar (20 y $10 \%$, respectivamente). De los ejemplares en los que no fue posible determinar el tamaño corporal, la mayor proporción se encontraba en el agua (3\%) (fig. 4).

\section{Discusión}

La estimación de la abundancia con base en las TE de los cocodrilos ha sido la principal herramienta de estimación y comparación utilizada en México (Sigler, Cedeño y Cupul, 2011), debido a que su uso representa un bajo costo (García-Grajales, Aguirre-León y Contreras-Hernández, 2007) y permite establecer las bases para la toma de decisiones en cuanto al concepto del uso sostenible (García-Grajales et al., 2007; Sánchez-Herrera, López, García-Naranjo y Benítez, 2011). 


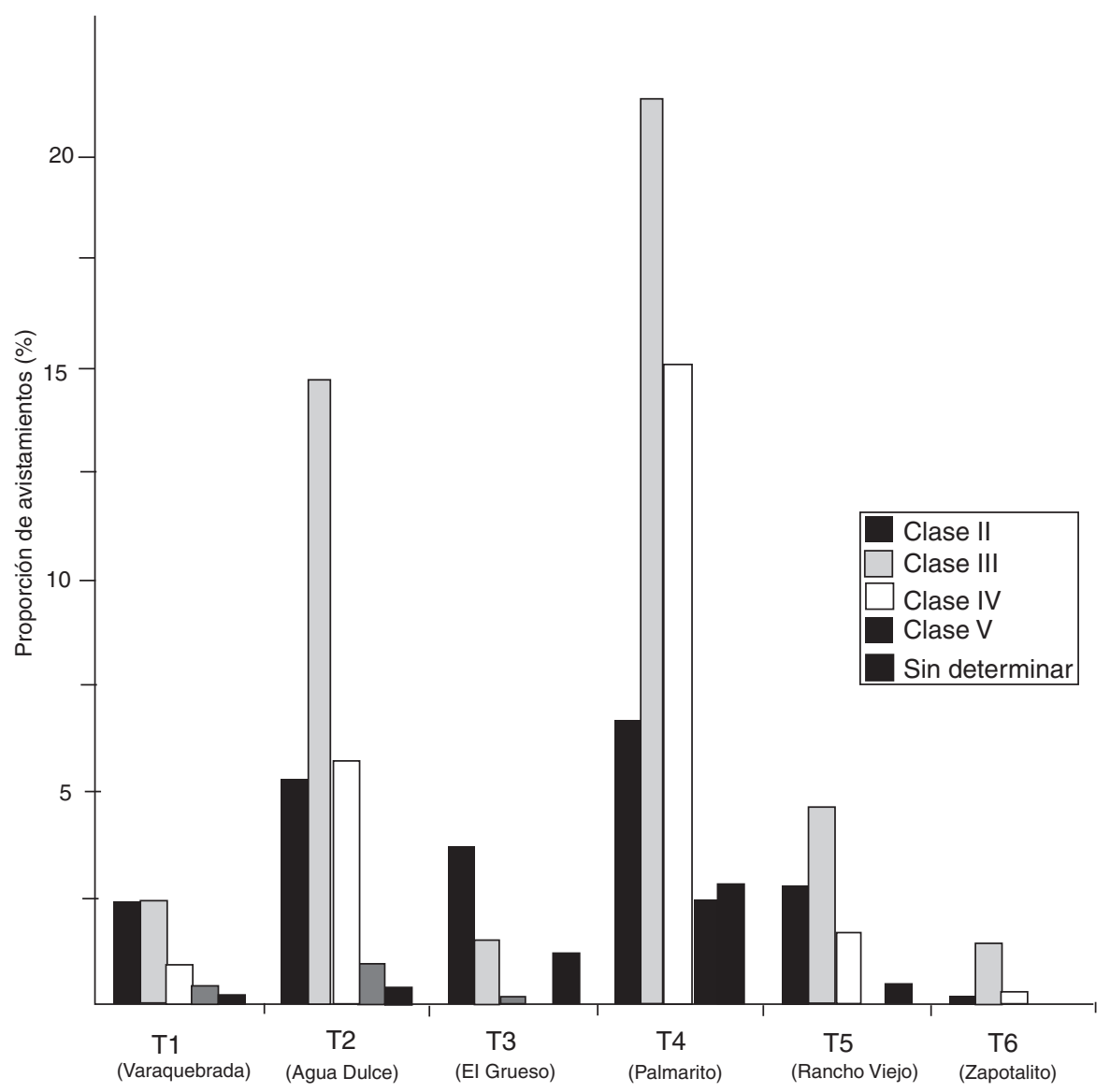

Figura 2. Estructura poblacional de cocodrilo americano (Crocodylus acutus) en los transectos recorridos dentro del Parque Nacional Lagunas de Chacahua, Oaxaca.

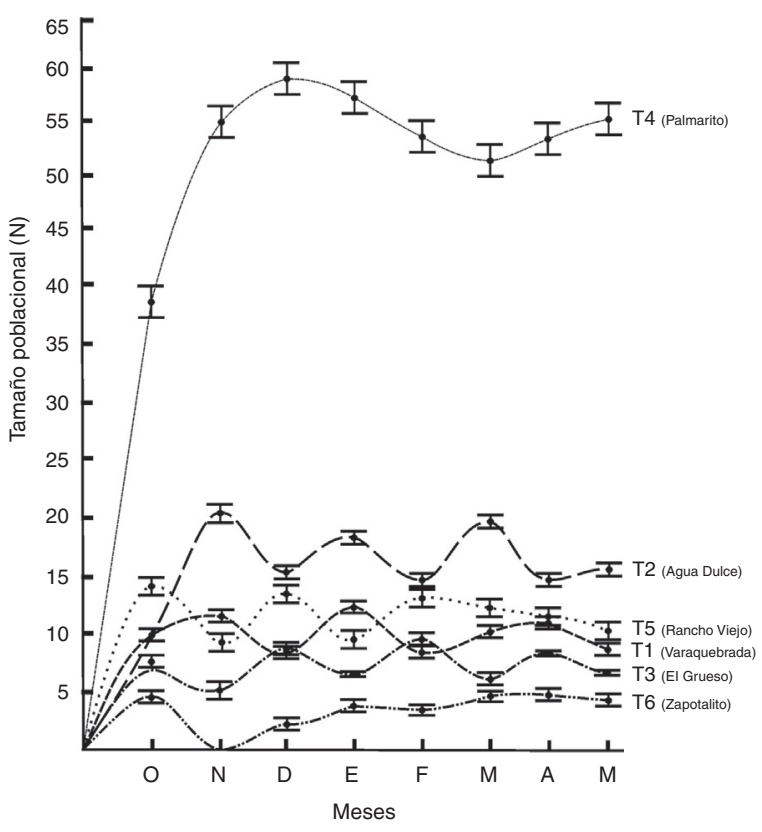

Figura 3. Fluctuación mensual del tamaño poblacional del cocodrilo americano (Crocodylus acutus) en los transectos establecidos del Parque Nacional Lagunas de Chacahua, Oaxaca.

Por muchos años, la estimación de la abundancia de las poblaciones de cocodrilos en México se basó exclusivamente en las TE de los individuos respecto a la longitud del área

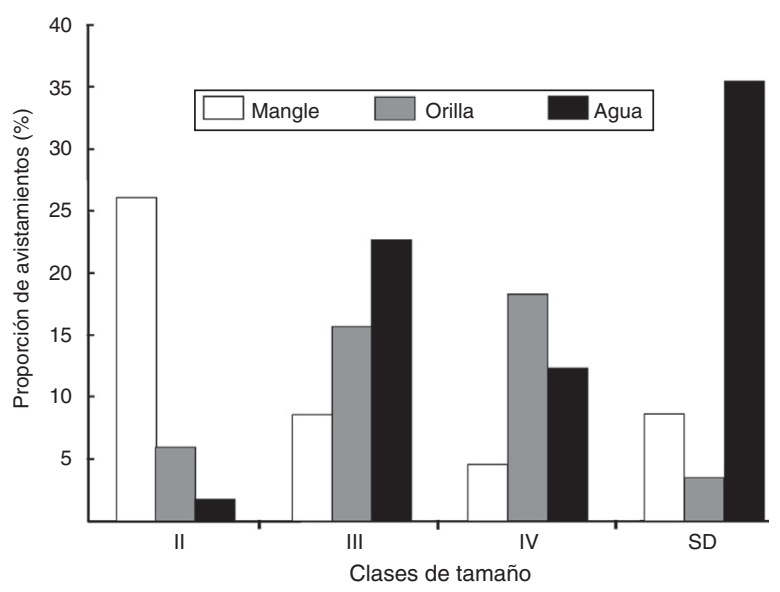

Figura 4. Frecuencia de uso por ambientes del cocodrilo americano (Crocodylus acutus) en el Parque Nacional Lagunas de Chacahua, Oaxaca.

muestreada, arrojando por consecuencia un índice de abundancia relativa, considerando el número de animales o sus rastros por unidad de esfuerzo (Ojasti y Dallmeier, 2000). Por tal situación, las TE se continúan efectuando de manera comparativa; sin embargo, la escala espacial sigue siendo el factor que permite establecer las comparaciones entre sitios. Para tal propósito, al comparar la TE obtenida en la laguna Palmarito con respecto a otras poblaciones en México se observó 
que la TE promedio obtenida (49 ind./km) es inferior a las TE registradas en la laguna Palmasola, Oaxaca (70.1 ind./km; García-Grajales y Buenrostro-Silva, 2014a), pero superior a las registradas en La Ventanilla, Oaxaca (47.33 ind./km; GarcíaGrajales y Espinosa-Reyes, 2001), la Encrucijada, Chiapas (2.1 ind./km; Martínez-Ibarra, Naranjo y Nelson, 1997) e incluso para algunas localidades de Jalisco (45.5 ind./km; HuertaOrtega, 2005) y Sinaloa (7.7 ind./km; Navarro-Serment, 2001); no obstante, las TE registradas en los restantes sitios (transecto) fueron numéricamente similares a las reportadas en los trabajos aquí señalados.

A pesar del inminente sesgo que existe en la estimación de la estructura poblacional por observación a distancia (Ron, Vallejo y Asanza, 1998), en la localidad de Palmarito y en general para todos los sitios, se observó que la estructura poblacional por tallas presentó una mayor abundancia de individuos de clase III (subadultos), seguido de individuos juveniles (clase II). Este tipo de estructura poblacional es poco común y discrepante a lo reportado para otras poblaciones en México, con la similitud de que los adultos sí aparecen con más frecuencia en la estructura de la población (Brandon-Pliego, 2007; Cupul-Magaña, Rubio-Delgado, Reyes-Juárez y Hernández-Hurtado, 2002). De manera similar a lo mostrado por García-Grajales y BuenrostroSilva (2014a), donde existe una mayor proporción de individuos subadultos (clase III) en la laguna Palmasola, infiriendo bajas tasas de mortalidad natural en la etapa juvenil con un consecuente reclutamiento al estadio subsecuente (Thorbjarnarson, 1989), en este trabajo también parece existir el reclutamiento a las siguientes etapas. Una razón probable de la estructura poblacional observada en este trabajo se puede atribuir a los efectos de conservación existentes en el ANP, proporcionando refugio en los manglares y cuerpos de agua de grandes dimensiones que permiten la existencia de hábitats disponibles y sitios intrincados de difícil acceso para el humano (Thorbjarnarson, 1989).

En cuanto a la frecuencia de uso por ambientes, la mayoría de los registros se obtuvieron en asociación con el manglar y con mayor proporción de uso por parte de los individuos de la clase II, lo que coincide con lo reportado por García-Grajales y Buenrostro-Silva (2014a) y Thorbjarnarson (1989), quienes mencionan que los cocodrilos frecuentemente prefieren permanecer escondidos en las raíces del mangle. Por otro lado, los ejemplares encontrados nadando en el agua fueron adultos reproductores y adultos, ya que solo estos tamaños corporales se aventuran a abandonar la seguridad que proporcionan las riberas cubiertas por manglar (Hernández-Vázquez, 2001).

Respecto a la abundancia mensual estimada, el canal Palmarito (PM) presentó la estimación más alta de todo el muestreo. Es posible asumir que el tamaño de población en este sitio no supera más de 50 organismos; sin embargo, cabe resaltar que la interconexión entre canales y lagunas aumenta la probabilidad de que más cocodrilos lleguen a este espacio debido a las características biológico-ambientales de la laguna Palmarito, como la alimentación, profundidad, temperatura y salinidad (Kushlan y Mazotti, 1989; Thorbjarnarson, 1989). Valdría la pena considerar, en futuros trabajos para este Parque Nacional, la posible correlación de la abundancia con respecto a la salinidad de los cuerpos de agua, ya que se ha demostrado que este parámetro es importante en la distribución de los cocodrilos, de manera que los organismos adultos soportan altos porcentajes de salinidad mientras que las tallas más pequeñas (subadultos y juveniles) se encuentran relegados a rangos de salinidad del 18 y $35 \%$ o (Richards, Mooij y de Angelis, 2004).

En cuanto a la estimación de las abundancias a través del modelo de Messel et al. (1981), pocos han sido los trabajos que han optado por generar la estimación a partir de la fracción visible de la población. Respecto a esto, Brandon-Pliego (2007) realizó un estudio donde estimó la abundancia de la población de la laguna La Palmita (Jamiltepec, Oaxaca) con un valor promedio superior (35.51 organismos) al obtenido en este estudio. Hernández-Hurtado, Romero-Villarruel y Hernández-Hurtado (2011) mostraron estimaciones de abundancia con intervalos superiores (68.1 a 148.02 organismos) en algunos transectos a las obtenidas en este trabajo, y con intervalos inferiores (1.31 a 12.3 organismos) en otros transectos. Recientemente, García-Grajales y Buenrostro-Silva (2014a) reportaron estimaciones de abundancia que fluctuaron de 32.7 a 93 individuos, siendo muy superiores a lo obtenido en este trabajo. Sin embargo, aunque la mayoría de estos trabajos reportan valores de abundancia superiores, un factor importante a considerar es la perspectiva de la escala espacial en las estimaciones poblacionales, es decir, las dimensiones espaciales de los cuerpos de agua y los transectos utilizados cuando son comparados entre sí; si bien los resultados mostrados por Hernández-Hurtado et al. (2011) son superiores en su mayoría a los registrados en este trabajo, las dimensiones espaciales entre los transectos de ambos sistemas (San Blas y Chacahua) son diferentes en términos de las longitudes, por lo que habría la posibilidad de que existieran diferentes condiciones en cuanto a las profundidades de los transectos, la vegetación e incluso las condiciones de salinidad.

El presente trabajo contribuye al conocimiento del estado poblacional de $C$. acutus en el PNLCh, donde el tamaño poblacional estimado parece mostrar valores altos para uno de los sitios evaluados con respecto a lo reportado en otras poblaciones del estado e incluso de la República Mexicana. Las causas por las cuales existe una población con valores representativos puede atribuirse a los efectos conservacionistas implicados en la denominación del ANP; sin embargo, dada la importancia de este recurso natural y su estatus de protección dentro de la Norma Ecológica 059 (Diario Oficial de la Federación, 2011), es imperante establecer un programa de monitoreo de largo plazo que permita conocer la tendencia de la población de cocodrilos en este sitio en particular. Aunado a esto, la existencia de grupos comunitarios capacitados y con experiencia en la ejecución de conteos nocturnos y manejo de cocodrilo debe ser una iniciativa que no debe dejarse en el olvido respecto a las actividades de manejo del PNLCh, y se sugiere fomentar el continuo empleo de estos grupos para realizar las acciones de monitoreo a largo plazo. Las primeras aproximaciones sobre la ecología poblacional y el estado actual del cocodrilo americano en el PNLCh son proporcionadas en este trabajo y representan la base del monitoreo poblacional en los años futuros para la administración de esta ANP. 


\section{Agradecimientos}

Los autores agradecen a la Universidad del Mar el apoyo logístico proporcionado y a Ignacio Carrasco Escobar (Gestión 2011-2014) del Parque Chacahua su apoyo para la gestión del recurso PROCODES de la Conanp y las distintas facilidades otorgadas en la estación de campo. Agradecemos a Oscar Hernández Bello (QEPD), Santa Ana Mariche, Giovanni Domínguez, Arnulfo Hernández, Jonhatan Marcial Mariche y Alejandro Domínguez, por su ayuda en las actividades de campo de este trabajo. Finalmente, a dos revisores anónimos que contribuyeron a mejorar este trabajo.

\section{Referencias}

Anónimo. (2000). Proyecto para la conservación, manejo y aprovechamiento sustentable de los Crocodylia en México. México, D.F.: Instituto de Ecología, Secretaría de Medio Ambiente y Recursos Naturales y Pesca.

Brandon-Pliego, J. D. (2007). Estudio poblacional de Crocodylus acutus (Cuvier, 1807) (Reptilia: Crocodylia) en Jamiltepec, Oaxaca. Ciencia y Mar, 11, 29-37.

Cupul-Magaña, F. G., Rubio-Delgado, A., Reyes-Juárez, A. y HernándezHurtado, H. (2002). Sondeo poblacional de Crocodylus acutus (Cuvier 1807) en el estero Boca Negra, Jalisco. Ciencia y Mar, 6, 45-50.

Cupul-Magaña, F. (2009). ¡A contar cocodrilos! Comentarios básicos sobre algunos métodos para evaluar poblaciones silvestres. Ciencia y Mar, 13, 3-14.

Chabreck, R. H. (1966). Methods of determining the size and composition of alligators populations in Louisiana. Proceedings 20 ${ }^{\text {th }}$ Annual Conference Southeastern Association of Game and Fish Commissioners, 20, 105-112.

Diario Oficial de la Federación. (2011). Norma Oficial Mexicana NOM-059SEMARNAT-2010, que determina las especies de flora y fauna silvestres terrestres y acuáticas, endémicas, amenazadas, en peligro de extinción y sujetas a protección especial. México, D.F.: Órgano del Gobierno Constitucional de los Estados, Gobierno Federal.

Ernst, C. H., Ross, F. D. y Ross, C. A. (1999). Crocodylus acutus (Cuvier) American crocodile. Catalogue American Amphibians and Reptiles, 700, $1-17$.

García, E. (1988). Modificaciones al sistema de clasificación climática de Köpen, para adaptarlo a las condiciones de la República Mexicana. México, D.F.: Offset Larios, S.A.

García-Grajales, J., Aguirre-León, G. y Contreras-Hernández, A. (2007). Tamaño y estructura poblacional de Crocodylus acutus (Cuvier 1807) (Reptilia: Crocodylidae) en el estero La Ventanilla, Oaxaca, México. Acta Zoológica Mexicana (nueva serie), 23, 53-71.

García-Grajales, J. y Buenrostro-Silva, A. (2013). New record of non-fatal attack by an American crocodile and geographic analysis of historical attacks in Oaxaca State, Mexico. Crocodile Specialist Group Newsletter, 32, 14-15.

García-Grajales, J. y Buenrostro-Silva, A. (2014a). Abundancia y estructura poblacional de Crocodylus acutus (Reptilia: Crocodylidae) en la laguna Palmasola, Oaxaca, México. Revista de Biología Tropical, 62, 165-172.

García-Grajales, J. y Buenrostro-Silva, A. (2014b). El Parque Nacional Lagunas de Chacahua, Oaxaca: perspectivas a sus 75 años. Revista Ciencia Ergo Sum, 21, 149-153.

García-Grajales, J. y Buenrostro-Silva, A. (2015). Áreas de interacción entre humanos y cocodrilos (Crocodylus acutus Cuvier) en Chacahua, Oaxaca, México. Revista Agroproductividad, 8, 25-33.

García-Grajales, J., Buenrostro-Silva, A. y Brandon-Pliego, J. D. (2008). Negative fatal interaction with American crocodile in Oaxaca, Mexico. Crocodile Specialist Group Newsletter, 27, 5-6.

García-Grajales, J. y Espinosa-Reyes, G. (2001). Densidad poblacional y estructura por tallas de la población del cocodrilo de río (Crocodylus acutus Cuvier 1807) en el estero de la Ventanilla, Oaxaca. En Anónimo (Ed.), Memorias de la Tercera Reunión de Trabajo del Subcomité COMACROM. (pp. 29-39). Culiacán: Cocodrilos Mexicanos, S.A. de C.V. /Semarnat.
García-Grajales, J. y López-Luna, M. A. (2010). Análisis bibliográfico del conocimiento de los cocodrilianos en México. Revista Latinoamericana de Conservación, 1, 25-31.

Hernández-Hurtado, H., Romero-Villarruel, J. J. y Hernández-Hurtado, P. S. (2011). Ecología poblacional de Crocodylus acutus en los sistemas estuarinos de San Blas, Nayarit, México. Revista Mexicana de Biodiversidad, 82, $887-895$

Hernández-Santos, I. (2009). Propuesta de programa para el manejo integral de la zona costera. Caso: Municipio de Villa de Tututepec de Melchor Ocampo, Oaxaca, México (Tesis de licenciatura). Puerto Ángel, Oaxaca, México: Universidad del Mar.

Hernández-Vázquez, S. (2001). Observaciones diurnas del cocodrilo de río Crocodylus acutus en el estero La Manzanilla, Jalisco, México. Boletín del Centro de Investigaciones Biológicas, 35, 283-294.

Huerta-Ortega, S. (2005). Dinámica poblacional del caimán (Crocodylus acutus, Cuvier 1807, Crocodylidae) en Jalisco, México (Tesis de maestría). Jalisco, México: Universidad de Guadalajara.

Kushlan, J. A. y Mazotti, F. J. (1989). Population biology of the American crocodile. Journal of Herpetology, 23, 7-21.

Marini, Z. F. (1999). Apropiación comunitaria y ordenamiento ecológico, principios de soberanía y sustentabilidad (Tesis de maestría). Oaxaca, México: Instituto Tecnológico Agropecuario de Oaxaca.

Martínez-Ibarra, J. A., Naranjo, E. y Nelson, C. (1997). Las poblaciones de cocodrilos (Crocodylus acutus) y caimanes (Caiman crocodilus) en una zona pesquera de la reserva de la biósfera 'La Encrucijada', Chiapas, México. Vida Silvestre Neotropical, 6, 21-28.

Mazzotti, F. J., Best, G. R., Brandt, L. A., Cherkiss, M. S., Jeffrey, B. M. y Rice, K. G. (2009). Alligators and crocodiles as indicators for restoration of Everglades ecosystems. Ecological Indicators, 9(6, Suppl.), S137-S149.

Messel, H., Vorlicek, G. C., Wells, A. G. y Green, W. J. (1981). Surveys of tidal river systems in Northern Territory of Australia and their crocodile populations. Monographs 1. Sydney: Pergamon Press.

Navarro-Serment, C. J. (2001). Uso de hábitat y conservación de Crocodylus acutus en el estero El Verde, Sinaloa. En Anónimo (Ed.), Memorias de la Tercera Reunión de Trabajo del Subcomité COMACROM. Culiacán, Sinaloa: Cocodrilos Mexicanos, S.A. de C.V. /Semarnat.

Ojasti, J. y Dallmeier, F. (2000). Manejo de fauna silvestre neotropical. Washington, DC: Smithsonian Institution, MAB Biodiversity Program.

Pérez-Delgado, P. (2002). Estado de conservación de la vegetación del Parque Nacional Lagunas de Chacahua: propuesta para su rehabilitación. En M. Alfaro y G. Sánchez (Eds.), Chacahua: reflejos de un parque. (pp. 21-38). Oaxaca: ONANP/PNUD/Semarnat/Plaza y Valdéz.

Ponce-Campos, P., Thorbjarnarson, J. y Velasco, A. (2012). Crocodylus acutus in IUCN 2012. IUCN the Red LIST of Threatened Species. Vers. 2012.2 [consultado 04 Mar 2016]. Disponible en: http://www.iucnredlist.org

Richards, P. M., Mooij, W. M. y de Angelis, D. L. (2004). Evaluating the effect of salinity on a simulated American crocodile (Crocodylus acutus) population with applications to conservation and Everglades restoration. Ecological Modelling, 180, 371-394.

Ron, S. R., Vallejo, A. y Asanza, E. (1998). Human influence on the wariness of Melanosuchus niger and Caiman crocodilus in Cuyabeno, Ecuador. Journal of Herpetology, 32, 320-324.

Sánchez-Herrera, O., López, G., García-Naranjo, A. y Benítez, H. (2011) Programa de monitoreo del cocodrilo de pantano (Crocodylus moreletii) México, Belice, Guatemala. México, D.F.: Comisión Nacional para el Conocimiento y Uso de la Biodioversidad /Secretaría de Medio Ambiente y Recursos Naturales.

Semarnat (Secretaría de Medio Ambiente y Recursos Naturales). (2014). Programa de manejo del Parque Nacional Lagunas de Chacahua. México, D.F. Comisión Nacional de Áreas Naturales Protegidas.

Sigler, L., Cedeño, J. R. y Cupul, F. (2011). Método de detección visual nocturna. En O. Sánchez, G. López, A. García-Naranjo, y H. Benítez (Eds.), Programa de monitoreo del cocodrilo de pantano (Crocodylus moreletii) México, Belice, Guatemala (pp. 105-127). México, D.F.: Comisión Nacional para el Conocimiento y Uso de la Biodiversidad, Secretaría de Medio Ambiente y Recursos Naturales.

Thorbjarnarson, J. (1989). Ecology of the American crocodile, Crocodylus acutus. En Anónimo (Ed.), Crocodiles, their ecology, 
management and conservation. Gland, Switzerland: IUCN Publications New Series.

Thorbjarnarson, J., Mazotti, F., Sanderson, E., Buitrago, F., Lazcano, M., Minkowski, K., et al. (2006). Regional habitat conservation priorities for the American crocodile. Biological Conservation, 128, 25-36.

Thorbjarnarson, J. T., Platt, S. y Saw-Tun-Khaing, U. (2000). A population survey of the estuarine crocodile in the Ayeryarwady Delta, Myanmar. Oryx, 34, 317-324.

Torres-Colín, R. (2004). Tipos de vegetación. En J. García-Mendoza, M. J. Ordoñez, y M. Briones-Salas (Eds.), Biodiversidad de Oaxaca (pp. 105-117). México, D.F.: Instituto de Biología, Universidad Nacional Autónoma de México/Fondo Oaxaqueño para la Conservación de la Naturaleza, World Wildlife Fund.

Venegas-Anaya, M., Escobedo-Galván, A. H., Balaguera, S., Lowrance, F., Sanjur, O. I. y Densmore, L. (2015). Population ecology of American crocodile (Crocodylus acutus) in Coiba National Park, Panama. Journal of Herptology, 49, 349-357.

Zar, J. H. (2010). Biostatistical analysis. New Jersey: Prentice-Hall. 